\title{
Self-Load Balanced Clustering Algorithm for Routing in Wireless Sensor Networks
}

\author{
Sivaraj Chinnasamy and Alphonse P J A \\ Department of Computer Applications, National Institute of Technology, Tiruchirappalli, 620015, INDIA \\ E-mail: sivamchinna@gmail.com, alphonse@nitt.edu \\ Janakiraman T N \\ Department of Mathematics, National Institute of Technology, Tiruchirappalli, 620015, INDIA \\ E-mail: janaki@nitt.edu
}

Received: 16 February 2017; Accepted: 10 April 2017; Published: 08 September 2017

\begin{abstract}
Energy-efficient routing is an extremely critical issue in unattended, tiny and battery equipped Wireless Sensor Networks (WSNs). Clustering the network is a promising approach for energy aware routing in WSN, as it has a hierarchical structure. The Connected Dominating Set (CDS) is an appropriate and prominent approach for cluster formation. This paper proposes an Energy-efficient Self-load Balanced Clustering algorithm (SLBC) for routing in WSN. SLBC has two phases: The first phase clusters the network by constructing greedy connected dominating set and the nodes are evenly distributed among them, using the defined parent fitness cost. The second phase performs data manipulations and new on-demand re-clustering. The efficiency of the proposed algorithm is analysed through simulation study. The obtained results show that SLBC outperforms than the recent algorithms like GSTEB and DGA-EBCDS in terms of network lifetime, CDS size, load dissemination, and efficient energy utilization of the network.
\end{abstract}

Index Terms-Wireless sensor networks, energyefficient routing, connected dominating set, load distribution, on-demand rotation.

\section{INTRODUCTION}

A Wireless Sensor Network is a collection of tiny electronic devices comprising sensors and transceivers. Sensors have the ability to monitor physical and environmental conditions. Typically, WSNs are unattended after deployment and the sensors have limitations on battery power, connectivity, storage and infrastructure [1]. The development of WSN was initiated by military applications (battlefield surveillance) [2]. WSNs are now implemented in commercial applications like human health monitoring, factory automation, environmental monitoring for temperature, lighting, humidity, and so on. Sensor networks have high reliability on scaling, as they support self-configuration and self-management [3]. In WSN, sensors' energy outage is seen as a major network failure, where sensors are the network's primary element and they are battery operated. Due to being unattended, recharging or replacing sensor's battery is very difficult and cost effective. So, designing energy-efficient communication structure in WSN is essential to prolong its lifetime. Among sensors' operations, data transmission is a major energy consumer and the consumption rate is directly proportional to the communication distance (distance between sender and receiver). So, lowering communication distance between nodes could increase the network lifetime.

Clustering the network is the key solution for nodes' energy conservation, as it has a hierarchical data collection mechanism. Clustering is a process of grouping sensors under a specific condition. A node from each group is selected as ClusterHead $(\mathrm{CH})$ to employ cluster operations. Constructing CDS in a network is an efficient solution for network clustering [4-8]. Some graph terminologies relevant to $\mathrm{CDS}$ construction are given below.

Graph: A graph $G$ is a pair of sets $(V, E)$, consists of a non-empty set $V$ of vertices (nodes) and another set $E$ whose elements are called edges (links), such that each edge $e$ is identified with an unordered pair $(v, u)$ of vertices.

Neighbour or adjacent node: Vertex $v$ is adjacent to $u$, if there is an edge $e$, such that $e=(u, v)$.

Neighbour Set $(N(v))$ : Neighbour set of $v$ is defined as $N(v)=\{u \mid(u, v) \in E\}$.

Degree: The degree of a node $v$ is the number of edges incident on $v$, and is denoted by $\operatorname{deg}(v)$.

Maximum degree $(\Delta)$ : The maximum degree of a node in graph $\mathrm{G}$ is $\Delta=\max \{\operatorname{deg}(v) \mid v \in V(G)\}$.

Dominating Set (DS): A set $D \subset V(G)$ a DS, if every node in $V-D$ is adjacent to at least one node in $D$. Nodes in DS are called dominators, and particularly known as ClusterHeads (CHs) in network clustering problems. Nodes of $V-D$ are called dominatees or 
cluster members.

Connected Dominating Set (CDS): If the graph induced by a subset DS is connected, then that DS is known as a Connected Dominating Set (CDS).

CDS size: The number of nodes in a CDS represents its size.

In CDS based clustering, each node in a CDS represents a $\mathrm{CH}$ that responsible for collecting data packets from cluster members, aggregate and transmit it to the base station [8]. Transmission rate depends on the number of CHs, so clustering a network with an optimal CDS size is essential to maximize the network lifetime. When CDS size is minimum, transmission rate decreases considerably. But, CHs suffer due to high data processing overheads that leading to rapid energy depletion. When CDS size is maximum, transmission rates increase extremely, thereby voiding the clustering benefits. So, this paper proposes a greedy CDS based clustering algorithm SLBC to construct an optimal number of clusters. The performance of the proposed algorithm is evaluated through simulation and compared with minimum sized CDS algorithm DGA-EBCDS and the maximum sized CDS algorithm GSTEB.

The rest of the paper is organized as follows: Section II reviews the related work. The network and radio model of the proposed algorithm is discussed in Section III. Section IV describes the problem statements and the proposed algorithm, SLBC. Section V presents the results and discussions of SLBC. Finally, Section VI concludes the paper.

\section{RELATED WORK}

Many heuristic CDS based clustering algorithms are proposed in literature with different design perspectives [4 - 6], [9-11]. Typically, WSNs are modelled as a Unit Disk Graph (UDG). In such graphs, nodes are unit circles, where any two nodes are said to be adjacent, if and only if, they reside within the unit distance. As data transmission is a major energy consumer in WSNs, most current algorithms address the Minimum sized CDS (MCDS) to reduce global communication distances and transmission rates [12].

Typically, CDS construction algorithms are classified [13] into centralized and decentralized (distributed) algorithms. Centralized algorithms prefer a MCDS with higher performance ratio. Such algorithms generally need total knowledge of network topology. In 1998, two centralized greedy heuristic algorithms for a general graphs were proposed by Guha and Khullar [7]. The first algorithm constructs a spanning tree (T) by choosing a maximum degree vertex as root. In each step, an additional fitted-vertex is added into $\mathrm{T}$. It has a performance ratio of $2(H(\delta)+1)$, where $\delta$ is maximum node degree. The second algorithm is an enhancement of the first and has two phases. The first phase constructs the dominating set and is connected using the Steiner Tree in the second phase. The performance ratio of the second algorithm is $H(\delta)+2$. Later Ruan et al., [14] proposed a one-step greedy approximation algorithm, which modifies the potential function of Guha et al., and obtains a performance ratio of $(2+\ln \delta)$. Misra et al. [15] presented a collaborative cover heuristic CDS construction algorithm that builds a Maximal Independent Set (MIS) using an effective coverage metric. Further, MIS nodes are connected as a Steiner tree by identifying Steiner nodes. This algorithm has a performance ratio of $(4.8+\ln 5)|\mathrm{opt}|+1.2$, where $|\mathrm{opt}|$ is the size of any optimal CDS in the network.

Das et al. [16] proposed a Steiner tree based algorithm called Pseudo-dominating Set Construction And Steiner Tree Spanning (PSCASTS), that builds the CDS in three stages. In the first stage, PSCASTS greedily constructs a pseudo-dominating set offering MIS with minimum cardinality. The second stage forms a Steiner tree by choosing Steiner nodes that connect more MIS nodes. Finally, in the third stage redundant CDS nodes are pruned. PSCASTS retains a performance ratio of collaborative cover heuristic proposed in [15]. Another Energy efficient MCDS construction algorithm (EMCDS) proposed by Tang, Qiang, et al. [17], constructs the CDS in two stages: the CDS construction stage and the pruning stage. Further CDS construction divided in to CS construction stage and DS construction stage. Dominators are selected in CS stage and the remaining uncovered nodes by CS stage is covered by DS stage. In pruning stage, E-MCDS removes the redundant dominators in CDS that reduces the CDS size. As, finding MCDS in UDG is a NP-hard problem [18] and constructs the cluster with more members, the lifetime of the $\mathrm{CHs}$ is shortened due to its additional data processing. Therefore, centralized-CDS algorithms are unfeasible for use in conducting CDS in WSNs. Hence, distributed algorithms are a better option for CDS construction as they prefer the metric of CDS construction process instead of a small sized CDS [19]. A metric may be a transmission rate and node's proximity.

Aziz et al. [20] proposed a Single-Phase Single Initiator (SPSI) Algorithm for distributed energy aware CDS construction. SPSI is an enhanced form of MPRs (Multi Point Relays) heuristic approach proposed by Qayyum et al. [21] that aims to reduce the CDS size. In this approach, each dominator chooses a set of connectors from its first hop neighbours, thereby covering the maximum two-hop neighbours. The chosen connectors become CHs and continue connector's selection from its neighbours. So, nodes should be aware of their 2-hop neighbours. It has $O(3 \Delta C+3 \Delta)$ time and $O(n)$ message complexity, where $\Delta$ is the maximum degree of a node. As maximum degree node has higher probability on dominator selection, dominators in SPSI are suffer heavily due to early energy depletion. DGA-EBCDS algorithm similar to SPSI was proposed by Kui et al. [22] to reduce CDS size. It has two phases; the MIS is constructed in the first phase, and nodes in MIS are connected using connectors in the second phase. MIS construction is prioritized by the node's degree and 
residual energy. The connectors are chosen from the neighbours of MIS nodes, which have more residual energy and better connection between them. The algorithm chooses a maximum of two connectors to connect adjacent MIS nodes (i.e. CHs). In DGA-EBCDS network lifetime is achieved by choosing higher residual energy nodes as $\mathrm{CHs}$ and by constructing the data collection tree. However, it includes more intermediate nodes (connectors) that increase the transmission rate unnecessarily. It also fails to distribute the nodes among CHs. This leads to maximum degree nodes die earlier as it has higher residual energy when choosing it.

Han, Zhao, et al proposed a General Self-Organized Tree-Based Energy-Balance (GSTEB) routing protocol [23] to construct a cluster backbone. In each round, the base station selects a maximum energy node as root and other nodes are choose one of its neighbours as $\mathrm{CH}$, which is closer to the root. If there are no such neighbours, the node selects the root as its CH. GSTEB is discussed in two types of radio models. The first radio model implements a data fusion technique and only the root can directly communicate with the base station. In the second radio model, data cannot be fused and also more nodes can communicate directly with the base station. As GSTEB constructs multi-branch with a chain like clustering, node's energy consumption is significantly increased, due to higher data reception and transmission, specifically the nodes that closer to the root.

Due to additional data manipulation, $\mathrm{CHs}$ premature death is a prominent issue in clustering algorithms. Recent algorithms to offset this, periodically perform reclustering. But, recurrent re-clustering generates additional control message overheads, leading to nodes unnecessary energy depletion. During re-clustering, data transmission is suspended, which is a substantial issue in time critical applications. This paper constructs wellbalanced CDS for network clustering and proposes an ondemand rotation scheme for re-clustering.

\section{NETWORK AND RADIO MODEL}

The proposed network model adopts some of the following assumptions.

The ' $n$ ' number of homogeneous and static sensor nodes are distributed in a two dimensional plan with compatible density, to ensure maximum connectivity and coverage. So, sensors have same transmission and sensing capability and the maximum transmission range of a node is denoted as $R$. Nodes are identified using a sequence of numbers $(1,2 \ldots n)$ called node ID. Nodes have a unique battery source with same amount of energy (in joule) during installation. It is assumed that the network is unattended and primary node failure is due to power depletion. Nodes are capable of dynamic transmission range adjustments. When two nodes are neighbours, they must reside within each other's transmission range $(R)$. Nodes are unaware of the geometrical information of their neighbours. But, it can compute the approximate distance of neighbours using
Received Signal Strength Indicator (RSSI) [24].

The proposed algorithm uses the first order radio model as in $[22,23,25]$ where radio transmitter and receiver circuitry energy consumption are $E_{\text {elec }}=50 \mathrm{~nJ} /$ bit and transmitter amplifier dissipate is considered $10 \mathrm{pJ} / \mathrm{bit} / \mathrm{m}^{2}$ for the free space propagation model $\left(\varepsilon_{f_{s}}\right)$ ( $d^{2}$ path loss) and $0.013 \mathrm{pJ} / \mathrm{bit} / \mathrm{m}^{4}$ for the tworay ground model ( $\left.\varepsilon_{m p}\right)$ ( $d^{4}$ path loss). Thus, $l$ bit message over distance $d$, energy consumption to transmit $\mathbf{E}_{\mathrm{TX}}(l, d)$ is calculated as

$$
E_{T X}(l, d)=\left\{\begin{array}{l}
l \cdot E_{\text {elec }}+l \cdot \varepsilon_{f s} \cdot d^{2}, \text { if } d \leq 1 \\
l \cdot E_{\text {elec }}+l \cdot \varepsilon_{m p} \cdot d^{4}, \text { if } d>1
\end{array}\right.
$$

Energy consumption to receive this message is

$$
E_{R X}(l)=l . E_{\text {elec }}
$$

Communication between two nodes is undertaken through IEEE 802.15.4 standard wireless radio full duplex method.

\section{Proposed Slbc Algorithm}

The proposed SLBC algorithm is described Setup and steady state phases. The setup phase describes weighted CDS construction and the steady state phase describes data collection and the proposed on-demand rotation scheme.

\section{A. Weight $(W)$ calculation}

Nodes weight calculation to become a $\mathrm{CH}$ is based on three properties; Reduced Degree $\left(\operatorname{deg}_{r}\right)$, coverage Integrity $(I)$ and Energy cost $\left(E_{C}\right)$.

Reduced Degree ( $\operatorname{deg}_{r}$ ): The reduced degree of a node represents the number of its uncovered neighbours. This property is adopted from $\mathrm{CH}$ selection criteria of the current algorithm [7]. Let $S$ be a set of nodes dominated by current CDS. If $N(v)$ is a neighbour set of node $v$, then the reduced degree of $v$ is calculated as:

$$
\operatorname{deg}_{r}(v)=|\{u: u \in N(v), u \notin S\}|
$$

Selecting the maximum reduced degree node's as a $\mathrm{CH}$, reduces CDS size considerably, as it forms cluster with more uncovered nodes.

Coverage Integrity ( $I$ ): Coverage integrity is a measurement to what degree a node can dominate the uncovered neighbours of its co-members. The Higher precedence of nodes coverage integrity in $W$ estimation increases the node's dissemination efficiency. Hence, CH's premature death caused by communication overheads is reduced considerably. Nodes, coverage 
integrity increases the probability of sensed data within cluster members being highly correlated, thereby increasing efficiency of data aggregation. It also reduces the energy consumption of $\mathrm{CHs}$, due to complex data aggregation processes. But, it could include additional nodes in the CDS, when a node with more coverage integrity and lesser reduced degree than competing nodes is chosen. Let node $v$ be dominated by $u$, then coverage integrity of $v$ with respect to $\mathrm{u}$ is defined as

$$
I(v, u)=\left|N(v) \cap\left\{\bigcup_{i \in N(u)}\{k: k \in N(i), k \notin S\}\right\}\right|
$$

where $N(v)$ and $N(u)$ are a neighbour set of $v$ and $u$ respectively.

Energy $\operatorname{Cost}\left(E_{C}\right)$ : Let $E_{r}(v)$ be the residual energy of node $v$ at round $r$ and $E_{i}(v)$ its initial energy. Then the energy cost of $v$ is defined as

$$
E_{C}(v)=\frac{E_{r}(v)}{E_{i}(v)-E_{r}(v)}
$$

Energy Cost of nodes lead to choosing a $\mathrm{CH}$ with maximum energy. Let $u$ be the $\mathrm{CH}$ of node $v$. Using equations (3), (4), and (5), weight of $v$ to become a $\mathrm{CH}$ is formulated with respect to $u$ as

$$
W(v, u)=\left(\alpha \times \operatorname{deg}_{r}(v)\right)+(\beta \times I(v, u))+\left(\gamma \times E_{C}(v)\right)
$$

$\alpha, \beta$ and $\gamma$ are balancing factors $(\alpha+\beta+\gamma=1)$, and are valued based on application perspectives. Weight estimation of the proposed SLBC algorithm does not consider the distance between CHs. The primary objective of SLBC is to construct an optimal CDS and so chooses a $\mathrm{CH}$ with more uncovered neighbours. An attempt is made to reduce the distance of $\mathrm{CHs}$ that increase $\mathrm{CDS}$ size as a node closer to $\mathrm{CH}$ will have minimum chance of having more uncovered neighbours.

\section{B. Parent Fitness cost}

As nodes can have more $\mathrm{CH}$ neighbours, SLBC disseminates them among clusters via an independent node distribution scheme, where nodes are distributed through use of a defined parent fitness cost. It is a CH's quality measurement estimated by a node using Euclidian distance and the approximated lifetime of the corresponding $\mathrm{CH}$. As data transmission consume much energy and as such consumption is directly proportional to the distance among nodes, decreasing transmission distance between the nodes and $\mathrm{CH}$ significantly lowers the nodes energy depletion [26]. So, the nodes are prepared towards the $\mathrm{CH}$ with minimum distance. Let $u$ be a $\mathrm{CH}$ and $v$ be a $\mathrm{CH}$ neighbour of $u$, residing in the communication path between $u$ and the base station. Let $d(u, v)$ be distance between $u$ and $v$. The estimated lifetime of $u$ is denoted as $T(u)$ and defined as

$$
T(u)=\frac{E_{r}(u)}{E_{T X}(l, d(u, v))+\left(E_{R X}(l) \times \operatorname{deg}_{r}(u)\right)}
$$

Consideration of the approximated lifetime of a $\mathrm{CH}$, aids energy efficient node distribution. Let $w$ belongs to $N(u)$, then the parent fitness value of $u$ regarding $w$ is defined as:

$$
F(u, w)=\frac{\lambda_{1}}{d(u, w)}+\left(\lambda_{2} \times T(u)\right)
$$

$\lambda_{1}, \lambda_{2}$ are balancing factors which lie between 0 and 1 , and whose sum is 1 .

\section{Clustering process}

The proposed algorithm uses depth first tree traversing mechanism with node colouring procedure to construct a CDS, where 'black', 'grey', and 'white' represent the $\mathrm{CHs}$ (dominators), cluster members and uncovered nodes respectively. A node is covered, it must be neighbour of a CDS node. All nodes are coloured 'white' when deployed. The base station selects a node from $r$ radius as root (initial $\mathrm{CH}$ ), say $u$, with maximum weight. Further, $u$ is added to the set CDS and coloured 'black'. When $u$ is selected as $\mathrm{CH}$, it broadcasts an elected message to neighbours with the following parameters; $\{I D$ $\left.\left.(u), E_{r}(u), \operatorname{deg}_{r}(u)\right)\right\}$. Let $v$ be a neighbour of $u$ that receives the message. Then it sends a response message to $u$ with the following parameters: $\{I D$ $\left.(v), E_{r}(v), J s \in\{0,1\}, W(v, u)\right\}$. Here, $J s$ notifies whether $v$ will join (1) the cluster of $u$ or not (0). It is defined as

$$
J_{S}= \begin{cases}1 & \operatorname{colour}(v) \rightarrow^{\prime} \text { white }^{\prime} \text { or if } F(v, u)>F(v, w) \\ 0 & \text { otherwise }\end{cases}
$$

After receiving the response from each neighbour, $u$ finalizes the cluster members according to the status of $J_{S}$ flag. The white neighbours of $u$ are coloured 'grey'. Subsequently, $u$ selects one of the 'grey' neighbour with maximum weight, say $v$, as a succeeding $\mathrm{CH}$ and is coloured black. Similar to $u, v$ sends an elected message to neighbours and forms a cluster according to the joining status of the neighbour's response. Further, white neighbours of $v$ coloured 'grey' and $v$ starts selection of the next $\mathrm{CH}$. This process continues until all the network nodes are coloured 'grey' or 'black'. A set of black nodes represents the CDS. Each CDS node form one cluster using its cluster members. After clustering, nodes reduce their transmission range according to the distance of its $\mathrm{CH}$. The procedure of the proposed algorithm is represented in algorithm 1 . The fitness based $\mathrm{CH}$ selection procedure is denoted as Independent Node Distribution (IND) and described in algorithm 2.

\section{Steady State Phase}

After successful clustering, a TDMA (Time Division 
Multiple Access) schedule is prepared by the base station. Further, nodes transmit their data to corresponding $\mathrm{CH}$ in a specified time slot. $\mathrm{CHs}$ collect data from cluster members, aggregate and transmit it to the base station directly (when the base station is a one-hop distance) or through intermediate $\mathrm{CHs}$ in the clustering hierarchy. One complete data collection by the base station is referred as a round. As premature death of $\mathrm{CHs}$ is a prominent issue in the design of clustering protocols, the current algorithms perform re-clustering at every round to overcome this. However, this recurrent re-clustering exponentially increases control message overheads for cluster formation, which shortens nodes lifetime significantly. During this phase, data transmission is interrupted, which is a precarious issue in time critical applications [27]. Therefore, SLBC proposes a new ondemand rotation scheme, which performs re-clustering when the residual energy of any $\mathrm{CH}$ is reduced to certain level of its cluster members.

On-demand rotation scheme: Let $u$ be a $\mathrm{CH}$ and $N_{d}(u)$ be a set of cluster members of $u$, then $u$ sends a special beacon message to the base station to initiate reclustering when the following condition is satisfied.

$$
\left(E_{I}(u)-E_{r}(u)\right) \leq \frac{\sum_{k \in N_{d}(u)} E_{r}(k)}{\left|N_{d}(u)\right|} \times \omega
$$

where $E_{r}(k)$ represents residual energy of $k$ at round $r$ and $\omega(0<\omega<1)$ is an energy threshold variable. As cluster members transmit sensed data to $\mathrm{CH}$, the current residual energy of cluster members is estimated based on the transmission cost. Let $r_{c}$ be the current round and $r_{i}$ be the round of the last successful (re-)clustering. Let $E_{i}(k)$ be the residual energy of $k$ at round $r_{i}, E_{r}(k)$ at $r_{c}$ which is calculated as

$$
E_{r_{c}}(k)=E_{i}(k)-\left(r_{c}-r_{i}\right) E_{T X}(k)
$$

$E_{T X}(k)$ is the transmission cost of one round. $E_{i}(k)$ is collected by $u$ when clustering. As nodes can find neighbours distance using the RSSI method, finding residual energy of neighbours does not need additional control messages. Whenever the base station receives this message from a $\mathrm{CH}$, it initiates re-clustering by performing the setup phase. The setup and steady state phases are sequentially executed till the first node fails. The performance of the proposed algorithm is evaluated through simulation and the results described in section $\mathrm{V}$.

\section{E. Energy consumption Analysis of proposed algorithm per round}

In each round, a node generates $l$ bit of data and send it to the $\mathrm{CH}$. Let $u$ be the $\mathrm{CH}$, consists of $n$ cluster members and $v_{i}$ be the $i^{\text {th }}(i \in\{1,2, \ldots n\})$ cluster member of $u$. Hence from Equation 1, the energy consumption for sending data from all the cluster members to $u$ is expressed as

$$
E_{c m}(u)=\sum_{i=1}^{n} l \times E_{\text {elec }}+l \times \varepsilon_{f s} \times\left(d\left(u, v_{i}\right)\right)^{2},
$$

Also from Equation (2), energy consumption of $u$ for receiving and aggregating cluster member's data is expressed as

$$
E_{C H}(u)=n\left(l \times E_{\text {elec }}\right)+(n+1)\left(l \times E_{D A}\right),
$$

where $E_{D A}$ represents the energy consumption to aggregate one bit of data. Since $u$ also performs sensing operation, data of $u$ is included in data aggregation. From Equations (12) \& (13), total energy consumption for intra-cluster data collection is derived as

$$
E_{\text {Collect }}(u)=E_{c m}(u)+E_{C H}(u),
$$

Let $l_{1}$ be the aggregated data length of $u$ and $f_{\text {agg }} \in(0,1)$ is the data aggregation factor [24]. Then $l_{1}$ is calculated as

$$
l_{1}=\frac{l \times(n+1)}{\left(n \times f_{\text {agg }}\right)+1}
$$

Further, $u$ sends aggregated data to the base station through a multi-hop model. Let $h$ be the hop distance of path from $u$ to the sink in the proposed clustering structure. Then the total energy depletion for sending this $l_{1}$ bit of data to the sink is calculated as

$$
E_{m_{-} h o p}(u)=l_{1} \sum_{i=1}^{h} 2 E_{\text {elec }}+\left(\varepsilon_{m p} \times d_{\text {toHop }}^{4}\right)
$$

where $d_{\text {toHop }} \leq R$ represents distance between two adjacent CHs. Total energy consumption of the proposed algorithm per round is calculated from Equations (12), (14) and (16), and given as

$$
E_{\text {perRound }}=\sum_{i=1}^{|C D S|}\left[E_{\text {Collect }}\left(u_{i}\right)+E_{c m}\left(u_{i}\right)+E_{m_{-} h o p}\left(u_{i}\right)\right]
$$




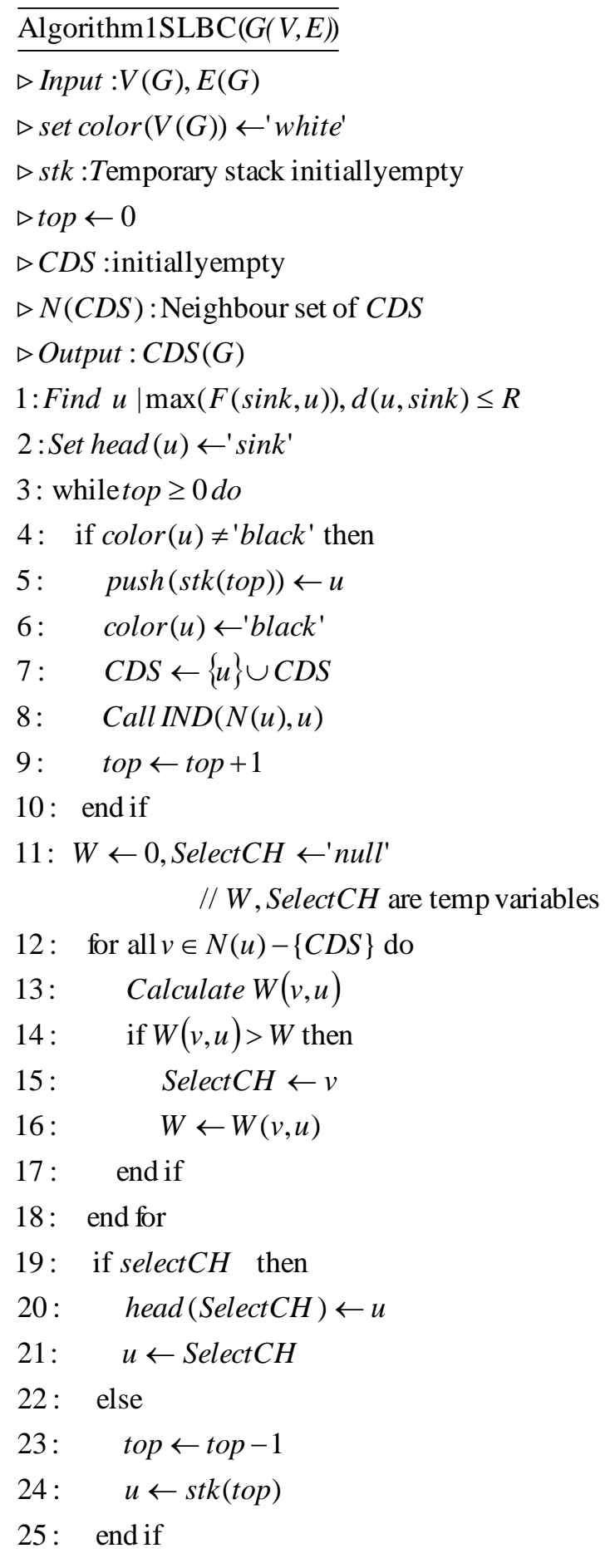

26: end while

\section{F. Time complexity of SLBC}

The time complexity of each stack operations is $\mathrm{O}(1)$. The proposed algorithm uses the backtrack method to construct the clusters that performs maximum of $|\mathrm{CDS}|$ times push and pop operations. Hence the time complexity of the while loop is $2|\mathrm{CDS}|$. The algorithm repeats the statement finding the new dominator in the maximum of $\Delta$ times inside the while loop. So the maximum time complexity of the proposed algorithm is $O(2 \Delta|C D S|)$.

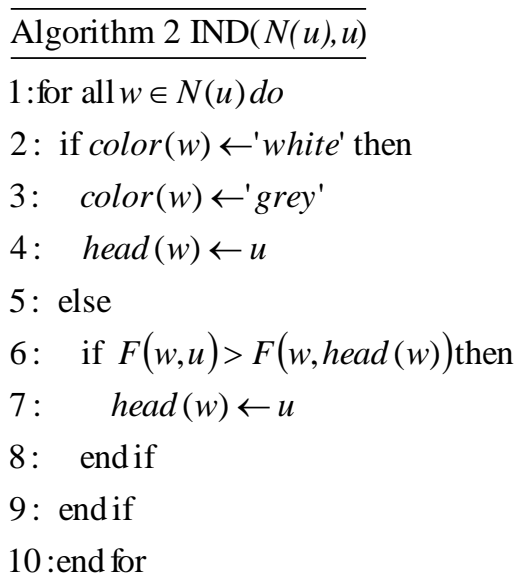

\section{EXPERIMANTAL RESULTS AND DISCUSSION}

This section discuss the experimental analysis of proposed and compared algorithms (E-MCDS [15], DGA-EBCDS [22] and GSTEB [23]) in various network scenarios and aspects.

\section{A. Simulation Setup}

This section describes the simulation results and performance evaluation of the proposed algorithm in terms of CDS size, load dissemination, network lifetime, and efficient energy utilization. The obtained results are compared to the current clustering algorithms E-MCDS, DGA-EBCDS and GSTEB. The aforementioned network model (in section III) is used to construct network topology for experiments. To ensure a unified comparison, the compared algorithms uses the similar network setup and energy model. Results are obtained from 100 experiments on each algorithm with node densities ranging from 50 to 200. Parameters used in the experiments are shown in Table 1. A node's initial transmission range is defined by Equation (18) as in [28], which estimates the minimum transmission range, thus ensuring full connectivity. Let $N$ be the number of nodes in a network and $L$ be a side of squared deployment region. The transmission range of a node is calculated as:

$$
R=\sqrt{2} L \times \sqrt{\frac{\log _{10} N}{N}}
$$

Table 1. Simulation Parameters And Their Values

\begin{tabular}{|c|c|}
\hline Network Deployment area Size & 100X100, 200X200 \\
\hline Number of nodes & 50 to 200 \\
\hline Base station/sink position & $\begin{array}{c}\text { Middle of the Area of } \\
\text { Interest }\end{array}$ \\
\hline Data Packet size & $4000 \mathrm{bits}$ \\
\hline Control packet size & $200 \mathrm{bits}$ \\
\hline Energy Consumption for TX,RX & $50 \mathrm{~nJ}$ \\
\hline $\begin{array}{c}\text { Energy for data aggregation } \\
\text { (EDA) }\end{array}$ & $5 \mathrm{~nJ} / \mathrm{bit} /$ signal \\
\hline Initial node energy & $2 \mathrm{Joule}$ \\
\hline Sensors Communication Range & $R$ \\
\hline
\end{tabular}




\section{B. Clustering Structure}

One of the main objectives of the proposed algorithm is to disseminate load among clusters. To show the superiority of the proposed algorithm on load dissemination, it is essential to evaluate the proposed cluster structure. Figures 1, 2, 3, and 4 show the sample clustering structure of the DGA-EBCDS, E-MCDS, GSTEB and proposed SLBC algorithm, respectively. DGA-EBCDS constructs CHs based on the node's degree and does not perform load distribution. Hence, DGAEBCDS suffers extremely due to uneven load dissemination of clusters, as shown in figure 1. As EMCDS use reduced degree of nodes, it shows better distributed structure than the DGA-EBCDS. However, EMCDS also fails to distribute the nodes among CHs. Thus, some CHs are suffered by higher cluster members, as shown in figure 2. The algorithm GSTEB constructs a tree structure based on the distance between the nodes and the base station. As branches are constructed like a chain of nodes, transmission rate and average communication distance between the nodes to the root increases significantly. But, the proposed SLBC algorithm employs IND process for distributing nodes among $\mathrm{CHs}$, which is proved in figure 4. As DGAEBCDS constructs the collection tree for data collection, average communication length of $\mathrm{CHs}$ in SLBC is slightly higher compared to DGA-EBCDS. But, this limitation is nullified when compared to the load dissemination improvement of the proposed algorithm.

\section{CDS size}

This section analyses the number of clusters constructed by E-MCDS, DGA-EBCDS and the proposed SLBC. Results are shown in figures 5 and 6, where figure 5 shows the CDS size obtained when the deployment area (L) is $100 \mathrm{~m}^{2}$ and figure 6 shows the CDS size of the algorithms, when $\mathrm{L}$ is $200 \mathrm{~m}^{2}$. GSTEB constructs chain like tree structure, if each non-leaf node in the tree is considered as a $\mathrm{CH}$, the number of clusters is enormous. So, GSTEB is not considered for CDS based studies.

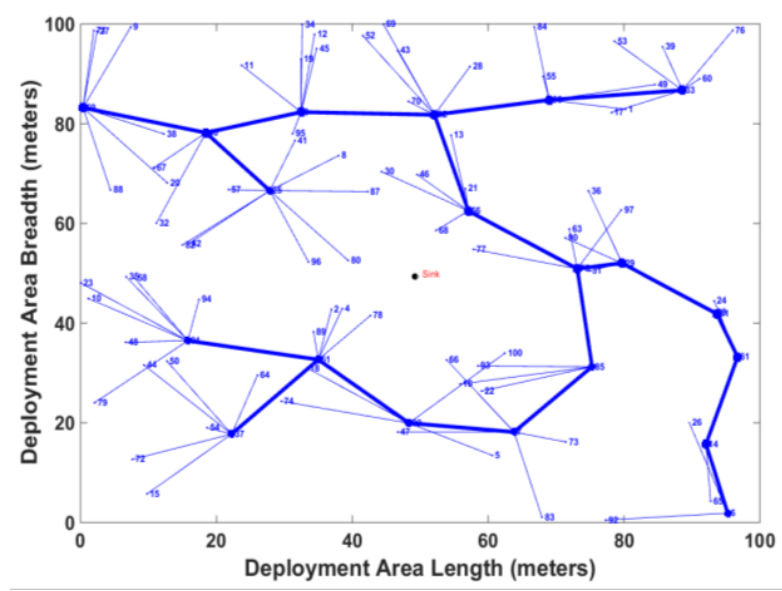

Fig.1. E-MCDS Clustering Structure

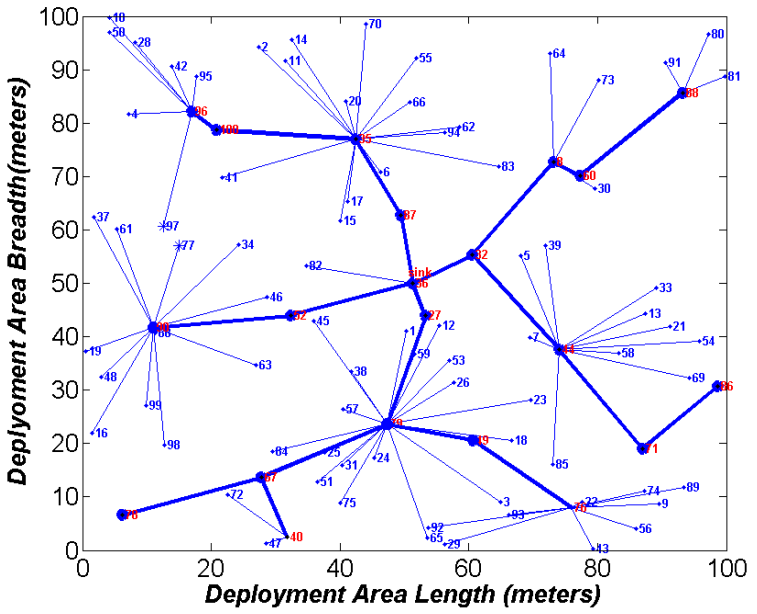

Fig.2. DGA-EBCDS Clustering Structure

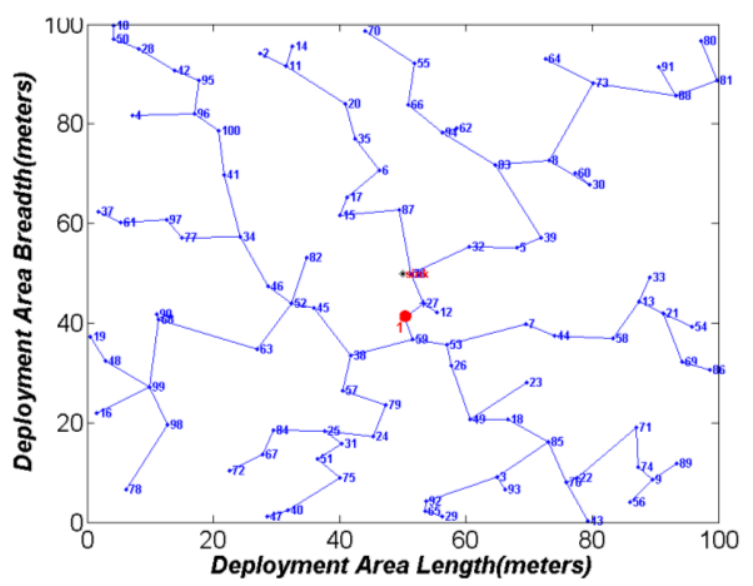

Fig.3. GSTEB Clustering Structure

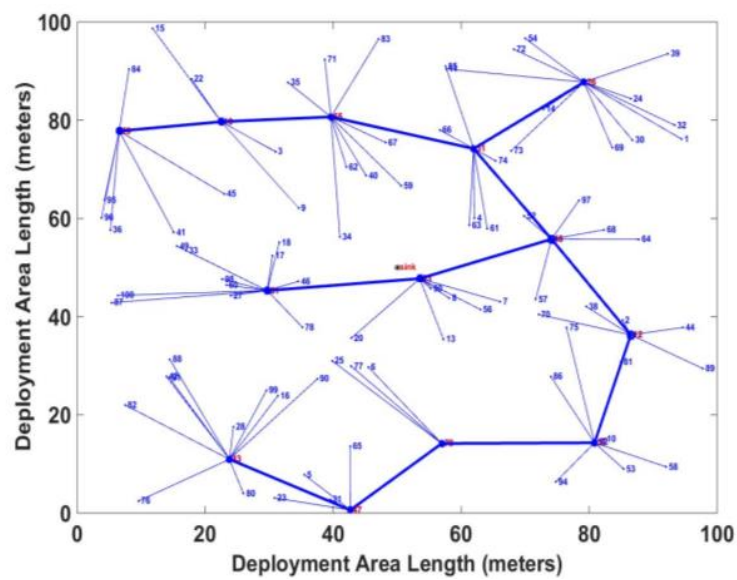

Fig.4. SLBC Clustering Structure

DGA-EBCDS selects higher degree nodes as CHs and connects those using common neighbours. Then, the size of CDS increases considerably due to more connecting nodes. However, as the proposed algorithm performs tree-based clustering, and as $\mathrm{CHs}$ are chosen based on nodes reduced degree, it ensures a smaller sized CDS than DGA-EDCDS, which is shown in figures 5 and 6. As like the proposed algorithm, E-MCDS also selects the $\mathrm{CHs}$ based on the reduced degree. But the DS 
construction stage of E-MCDS includes some more nodes into the CDS. Though, E-MCDS shows CDS size, which is very nearer to the proposed algorithm. As nodes transmission range is defined based on the network size and deployment area, nodes average degree is reduces when network size increases. Then the algorithms constructs more $\mathrm{CHs}$ to cover the entire network region. So, in figures 5 and 6 the size of CDS increases with network size and deployment region.

\section{Load dissemination}

Load dissemination of the proposed algorithm is evaluated based on the cluster density (number of nodes in a cluster) and size difference between maximum and minimum dense clusters. In each experiment, the number of nodes in the maximum dense cluster is obtained and the average of this result is used to analyses cluster density. The result is shown in figures 7 and 8 for two size of deployment areas $100 \mathrm{~m}^{2}$ and $200 \mathrm{~m}^{2}$ respectively. DGA-EBCDS selects maximum degree nodes as $\mathrm{CH}$ and does not perform node distribution among clusters. Hence, some CHs suffer by additional cluster members, where others have a minimum. In a worst case scenario, nodes in a dense cluster is equal to 30 percentage of network size, as depicted in figures 7 and 8. As E-MCDS selects the $\mathrm{CHs}$ based on the reduced degree, it reduced the number of nodes per $\mathrm{CH}$. But, it's not perform node distribution among $\mathrm{CHs}$, so still the $\mathrm{CHs}$ are suffered by uneven load dissemination.

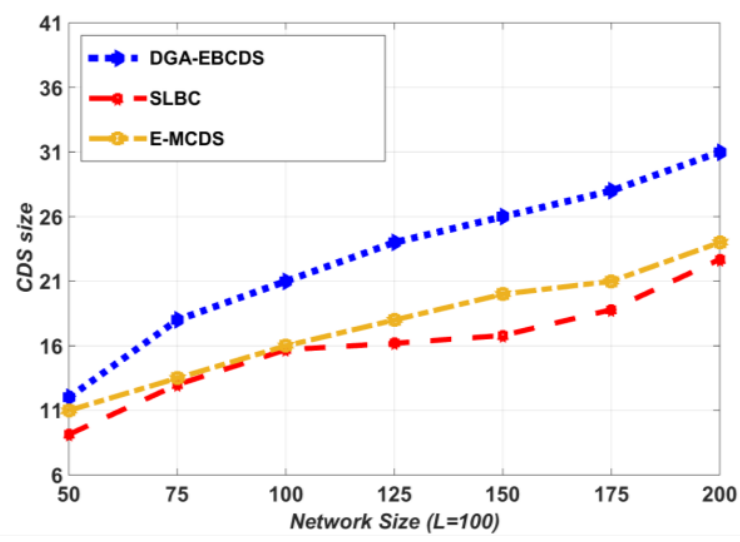

Fig.5. Comparison of CDS Size when $\mathrm{L}=100$.

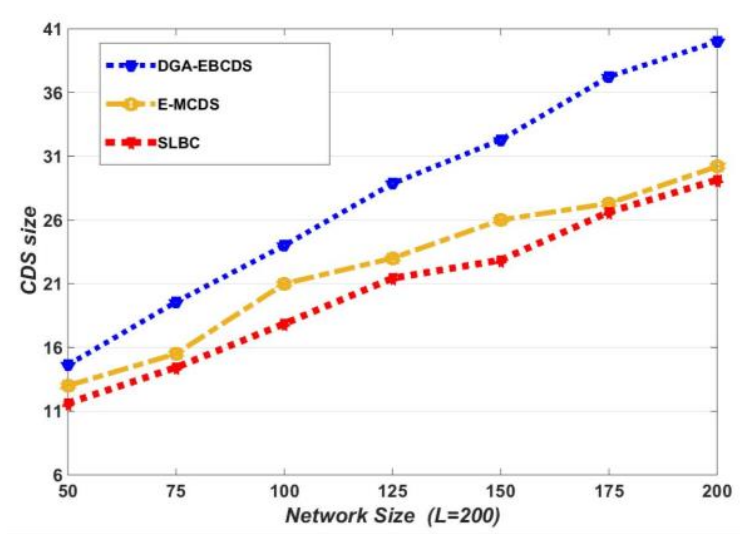

Fig.6. Comparison of CDS Size when L=200



Fig.7. Comparison of average cluster size $\left(\mathrm{L}=100 \mathrm{~m}^{2}\right)$

The proposed algorithm uses the coverage density of the nodes is one of the prime factor for $\mathrm{CH}$ selection, which increases the efficiency of the IND process. So, nodes are evenly distributed among CHs. In figures 7 and 8, the proposed algorithm constructs a cluster with minimum nodes than DGA-EBCDS and E-MCDS for all network sizes. Figure 9 shows the size difference between maximum and minimum dense clusters when $\mathrm{L}$ is $100 \mathrm{~m}^{2}$. Among the compared algorithms, the SLBC shows the least members difference between the maximum and minimum dense clusters. Hence, it is proved that the proposed algorithm is superior to DGA-EBCDS and EMCDS in terms of load dissemination.

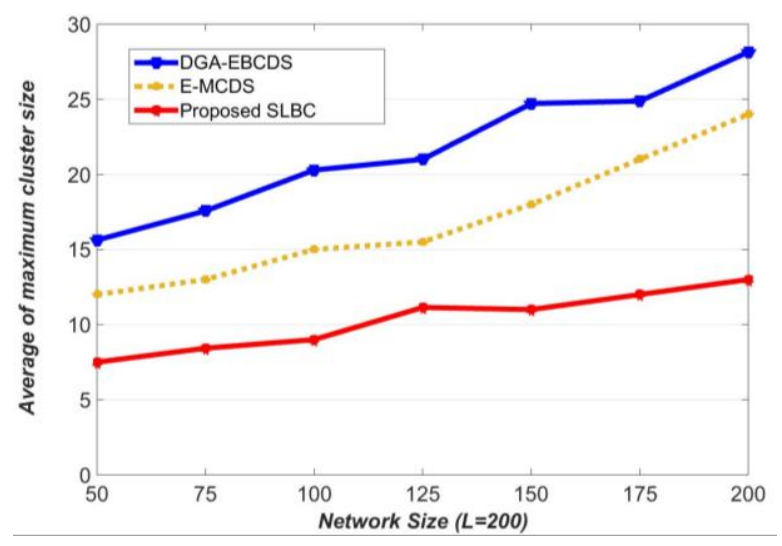

Fig.8. Comparison of average cluster size $\left(\mathrm{L}=200 \mathrm{~m}^{2}\right)$

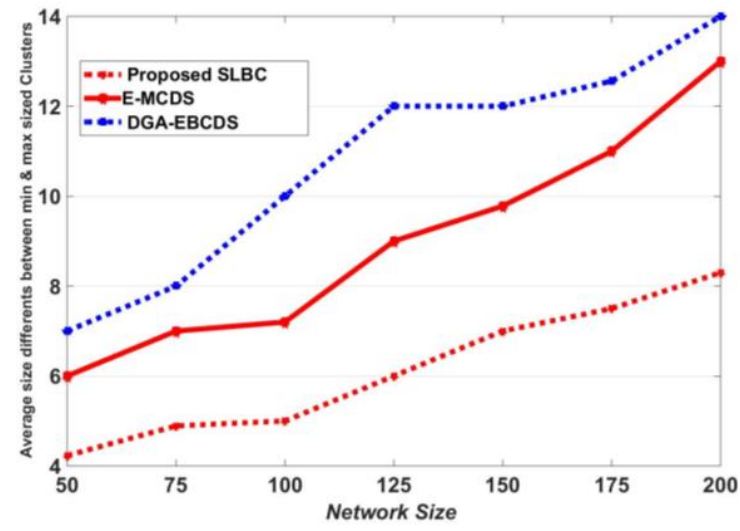

Fig.9. Comparison of average size different between $\min \& \max$ sized clusters

I.J. Intelligent Systems and Applications, 2017, 9, 46-57 




Fig.10. Average number of rotations by SLBC

\section{E. Re-clustering overheads of SLBC}

As the proposed algorithm performs on-demand rotation for avoiding early energy depletion of $\mathrm{CH}$, average number of rotations per experiment is considered for analysing its re-clustering overheads. Figure 10 shows the average rotations yielded by the proposed algorithm SLBC against various network sizes, when $L$ is $100 \mathrm{~m}^{2}$. As cluster density increases with network size, $\mathrm{CHs}$ experience higher energy depletion due to more data processing. So, the frequency of re-clustering is increases with the network size, as it is shown in figure 10. However, the percentage of rotations by SLBC against the number of its rounds is approximately $0.83 \%$, which infers the re-clustering overheads of SLBC is insignificant.

\section{F. Network Lifetime when first node dies}

Network lifetime is analysed in terms of rounds. One round represents a complete cycle of data transmission from all the nodes to the base station. The number of rounds is calculated from the initialization of the network till the first node dies. Figure 11 represents the comparison of network lifetime for the proposed and existing algorithms in various network sizes. The algorithm GSTEB builds a routing tree (Case1 in GSTEB) based on the distance between the nodes and the root node. So, almost all nodes in GSTEB participate in data forwarding, increasing the transmission rate exponentially. Due to more data transmission overheads, nodes experience undesirable energy depletion and energy is drained earlier. Moreover, the algorithm's reclustering fails to distribute nodes energy consumption, as it is just used to change data flow direction. Also, during re-clustering nodes suffer by control message overheads. These limitations of GSTEB reduce the network lifetime considerably.

In DGA-EBCDS, CHs are severely suffered by uneven load dissemination, so it's experience more data processing and die earlier. As DGA-EBCDS is not consider the intra-cluster communication distance for clustering, normal nodes have more probability to join with the far $\mathrm{CH}$. As a result, it spends more energy for data transmission. For these reasons, DGA-EBCDS shows minimum network lifetime when compared to the proposed SLBC and GSTEB. The algorithm E-MCDS is concentrates to reduce the transmission rate by reducing CDS size, then the CHs in E-MCDS is heavily suffered by higher data processing and die earlier. But, it reduces the density of cluster through reduced degree based $\mathrm{CH}$ selection procedure, so it shows better lifetime than DGA-EBCDS.

The proposed SLBC algorithm selects $\mathrm{CHs}$ based on the defined weight factors, which leads to select the $\mathrm{CHs}$ with more optimal and healthier. As SLBC distributes cluster members among adjacent $\mathrm{CHs}$ through IND procedure using defined parent fitness value, it distributes nodes energy consumption evenly. Also, nodes join the nearest $\mathrm{CH}$ using fitness based $\mathrm{CH}$ selection thereby reducing energy consumption significantly. Moreover, premature death of $\mathrm{CHs}$ is avoided by the proposed ondemand re-clustering method and also the control message overheads of this re-clustering method is claimed as insignificant (ref section $\mathrm{V}(\mathrm{E})$ ). Therefore, the proposed algorithm prolongs the network lifetime efficiently when compared with the existing algorithms DGA-EBCDS, E-MCDS and GSTEB, it is shown in figure 11. It is proved that the proposed algorithm SLBC shows outstanding performance in enhancing network lifetime and achieving the network lifetime more than $30 \%$ compared to GSTEB, more than $60 \%$ compared to E-MCDS and more than $200 \%$ compared to DGAEBCDS.

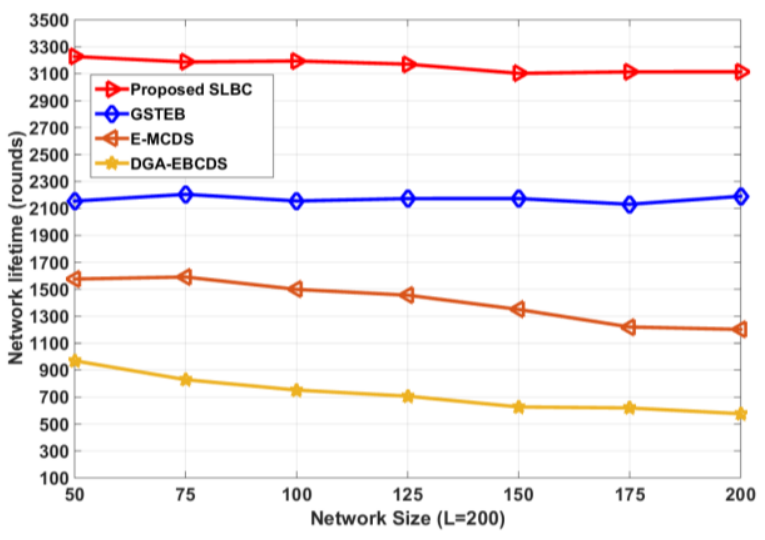

Fig.11. Comparison of network lifetime (First node dies)

\section{G. Network lifetime with respect to percentage of dead nodes}

As nodes have more remaining energy when the first node dies, this section analysis the network enhancement of algorithms until $30 \%$ of nodes are dead. Network size of this analysis is 200 nodes and the result shown in figure 12. According to figure 13, DGA-EBCDS unused maximum node's energy, due to uneven energy distribution. Using this unused nodes energy, it shows improved performance in estimating the lifetime against the percentage of nodes death. After $30 \%$ of nodes dead, DGA-EBCDS prolongs network lifetime nearly $200 \%$ more than after the death of the first node. GSTEB prolongs the network lifetime more than $35 \%$ from its achievement when the first node dies. The algorithm E- 
MCDS also increases the lifetime of network by $90 \%$ than its previous attainment. As, the proposed algorithm distributes nodes energy consumption evenly through energy aware node distribution (fitness based $\mathrm{CH}$ selection) and on-demand re-clustering method, the maximum node energy is used before the first node dies (as discussed in subsection $\mathrm{V}(\mathrm{E})$ ). So, it prolongs the network lifetime by $26 \%$ from its own attainment. However, the proposed algorithm shows the network lifetime more than $22 \%, 55 \%$ and $76 \%$ compared to GSTEB, E-MCDS and DGA-EBCDS respectively, when the $30 \%$ of nodes are dead.

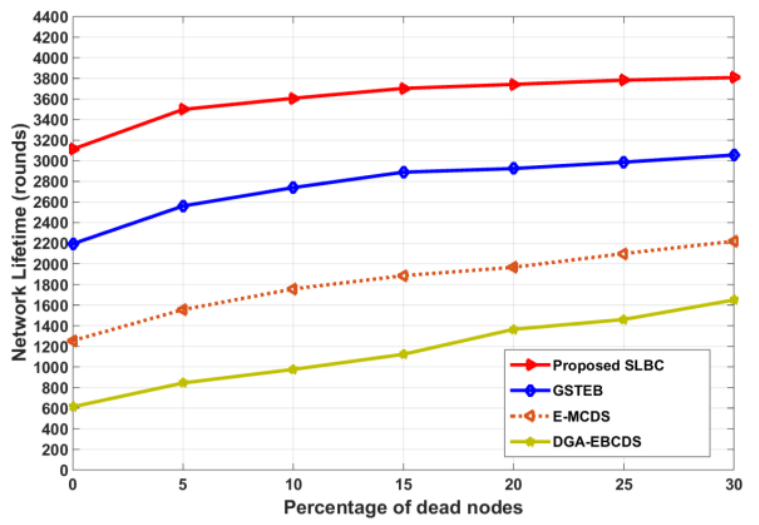

Fig.12. Comparison of network lifetime for percentage of dead nodes

\section{H. Energy utilization of the nodes}

Prolonging network lifetime through an efficient energy distribution among nodes is the primary objective of the proposed algorithm. When the algorithm succeeds in energy distribution, it utilizes more nodes energy to enhance the network lifetime. Nodes remaining energy at the end of each simulation is obtained and considered for the analysis of nodes energy utilization. The comparison of nodes average residual energy against network lifetime when the first node dies is shown in figure 13 and the comparison of energy utilization of nodes when the percentage of dead nodes is shown in figure 14. As DGAEBCDS suffers heavily due to uneven energy depletion issue, $\mathrm{CHs}$ die prematurely. So, it leaves more network energy unused. E-MCDS also fails to distribute the nodes among cluster, so $\mathrm{CHs}$ from dense cluster die earlier, leaving maximum network energy unemployed. Also nodes in GSTEB experience more data forwarding, especially those nearer to the root. This leads to shorter network lifetime, leaving significant amount of network energy unemployed. But, the proposed algorithm, avoids $\mathrm{CHs}$ premature death through on-demand rotation scheme with negligible re-clustering overheads and reduces the nodes data transmission energy cost through proposed IND process. Then, nodes energy consumption is efficiently distributed over the network. So, the proposed SLBC algorithm employs more nodes energy and significantly prolongs the network lifetime. The proposed SLBC algorithm is uses the network energy nearly $20 \%$ more than the immediate performer GSTEB achieving $30 \%$ of lifetime enhancement. It also proves that the proposed algorithm consumes least amount of nodes energy than the compared algorithms for one round of data gathering. Figure 13 and 14 infer that the proposed algorithm efficiently utilized the node's energy in order to enhance the network lifetime.



Fig.13. Comparison of energy utilization of nodes (first node dies)

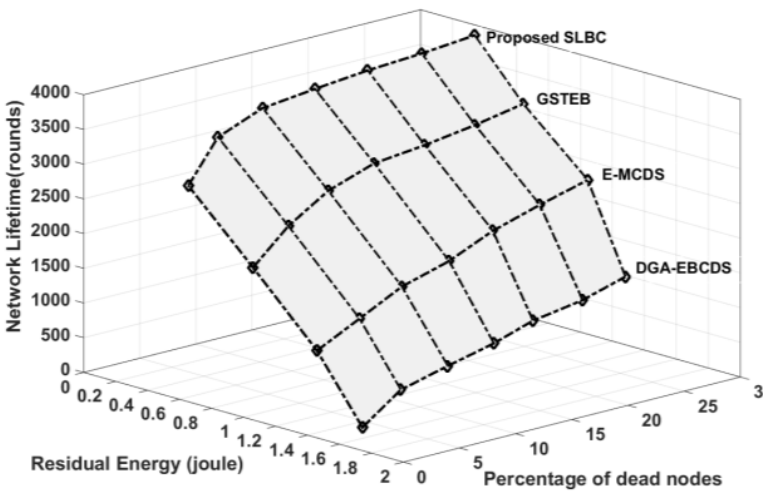

Fig.14. Comparison of energy utilization of nodes (when the percentage of dead nodes)

\section{CONCLUSION}

Efficient energy consumption of nodes is significant to prolong wireless sensor network lifetime, as it comprises power-constrained sensor nodes. In this paper, the algorithm SLBC is proposed to extend WSNs lifetime. SLBC extends WSNs lifetime by constructing the CDS with CHs being chosen based on a defined node's weight. During CDS selection, nodes are evenly distributed among $\mathrm{CHs}$, using an independent node distribution procedure. The proposed algorithm also evades control message overheads of re-clustering through an ondemand rotation scheme. Through an optimal CDS selection and even load distribution procedure, the proposed algorithm significantly prolongs network lifetime than the recent algorithms DGA-EBCDS, EMCDS and GSTEB. Simulations results show that SLBC achieves network lifetime more than 30\%, 55\% and $200 \%$ compared to the recent algorithms GSTEB, EMCDS and DGA-EBCDS respectively. 


\section{REFERENCES}

[1] Vamsi, P. Raghu, and Krishna Kant. "Self-adaptive trust model for secure geographic routing in wireless sensor networks." International Journal of Intelligent Systems and Applications 7.3 (2015): 21.

[2] Ashrafuddin, Md, Md Manowarul Islam, and Md Mamunor-Rashid. "Energy efficient fitness based routing protocol for underwater sensor network." International Journal of Intelligent Systems and Applications 5.6 (2013): pp.61-69.

[3] Khan, Koffka, and Wayne Goodridge. "Fault Tolerant Multi-Criteria Multi-Path Routing in Wireless Sensor Networks." International Journal of Intelligent Systems and Applications 7.6 (2015): 55.

[4] Liu, Yunhuai, Qian Zhang, and Lionel Ni. "Opportunitybased topology control in wireless sensor networks." IEEE Transactions on Parallel and Distributed Systems 21.3 (2010): 405-416.

[5] Wightman, Pedro M., and Miguel A. Labrador. "A3: A topology construction algorithm for wireless sensor networks." Global Telecommunications Conference, 2008. IEEE GLOBECOM 2008. IEEE. IEEE, 2008.

[6] Li, Deying, et al. "Minimum total communication power connected dominating set in wireless networks." International Conference on Wireless Algorithms, Systems, and Applications. Springer Berlin Heidelberg, 2012.

[7] Guha, Sudipto, and Samir Khuller. "Approximation algorithms for connected dominating sets." Algorithmica 20.4 (1998): 374-387.

[8] Younis, Ossama, and Sonia Fahmy. "HEED: a hybrid, energy-efficient, distributed clustering approach for ad hoc sensor networks." IEEE Transactions on mobile computing 3.4 (2004): 366-379.

[9] Dai, Fei, and Jie Wu. "An extended localized algorithm for connected dominating set formation in ad hoc wireless networks." IEEE transactions on parallel and distributed systems 15.10 (2004): 908-920.

[10] J. Wu and Cardei, "Extended dominating set and its applications in ad hoc networks using cooperative communication," Parallel and Distributed Systems, IEEE Transactions on, vol. 17, no. 8, pp. 851- 864, 2006.

[11] $\mathrm{Wu}, \mathrm{Jie}$, et al. "Extended dominating set and its applications in ad hoc networks using cooperative communication." IEEE Transactions on Parallel and Distributed Systems 17.8 (2006): 851-864.

[12] Cheng, Xiuzhen, et al. "A polynomial-time approximation scheme for the minimum-connected dominating set in ad hoc wireless networks." Networks 42.4 (2003): 202-208.

[13] Yu, Jiguo, et al. "Connected dominating sets in wireless ad hoc and sensor networks-A comprehensive survey." Computer Communications 36.2 (2013): 121-134.

[14] Ruan, Lu, et al. "A greedy approximation for minimum connected dominating sets." Theoretical Computer Science 329.1-3 (2004): 325-330.

[15] R Misra, Rajiv, and Chittaranjan Mandal. "Minimum connected dominating set using a collaborative cover heuristic for ad hoc sensor networks." IEEE Transactions on parallel and distributed systems 21.3 (2010): 292-302.

[16] Das, Ariyam, et al. "An improved greedy construction of minimum connected dominating sets in wireless networks." Wireless Communications and Networking Conference (WCNC), 2011 IEEE. IEEE, 2011.
[17] Tang, Qiang, et al. "An energy efficient MCDS construction algorithm for wireless sensor networks." EURASIP Journal on Wireless Communications and Networking 2012.1 (2012): 83.

[18] Clark, Brent N., Charles J. Colbourn, and David S. Johnson. "Unit disk graphs." Discrete mathematics 86.1-3 (1990): 165-177.

[19] Al-Nabhan, Najla, Mznah Al-Rodhaan, and Abdullah AlDhelaan. "Distributed energy-efficient approaches for connected dominating set construction in wireless sensor networks." International Journal of Distributed Sensor Networks (2014).

[20] Aziz, Azrina Abd, and Y. Ahmet Şekercioğlu. "A distributed energy aware connected dominating set technique for wireless sensor networks." Intelligent and Advanced Systems (ICIAS), 2012 4th International Conference on. Vol. 1. IEEE, 2012.

[21] Qayyum, Amir, Laurent Viennot, and Anis Laouiti. "Multipoint relaying for flooding broadcast messages in mobile wireless networks." System Sciences, 2002. HICSS. Proceedings of the 35th Annual Hawaii International Conference on. IEEE, 2002.

[22] X. Kui, Y. Sheng, H. Du, and J. Liang, "Constructing a CDS-based network backbone for data collection in wireless sensor networks," International Journal of Distributed Sensor Networks, vol. 2013, 2013.

[23] Han, Zhao, et al. "A general self-organized tree-based energy-balance routing protocol for wireless sensor network." IEEE Transactions on Nuclear Science 61.2 (2014): 732-740.

[24] Kui, Xiaoyan, et al. "Constructing a CDS-based network backbone for data collection in wireless sensor networks." International Journal of Distributed Sensor Networks 9.4 (2013): 258081.

[25] Benkic, Karl, et al. "Using RSSI value for distance estimation in wireless sensor networks based on ZigBee." Systems, signals and image processing, 2008. IWSSIP 2008. 15th international conference on. IEEE, 2008.

[26] Heinzelman, Wendi Rabiner, Anantha Chandrakasan, and Hari Balakrishnan. "Energy-efficient communication protocol for wireless microsensor networks." System sciences, 2000. Proceedings of the 33rd annual Hawaii international conference on. IEEE, 2000.

[27] Santi, Paolo, and Douglas M. Blough. "The critical transmitting range for connectivity in sparse wireless ad hoc networks." IEEE transactions on Mobile Computing 2.1 (2003): 25-39.

[28] Li, Jianpo, Xue Jiang, and I-Tai Lu. "Energy Balance Routing Algorithm Based on Virtual MIMO Scheme for Wireless Sensor Networks." Journal of Sensors (2014):1-7

\section{Authors' Profiles}

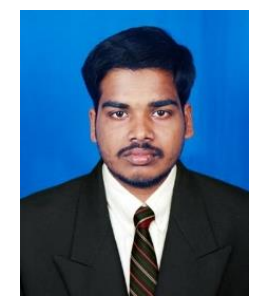

Mr. Sivaraj C is a $\mathrm{PhD}$ scholar in the Department of Computer Applications at National Institute of Technology (NITT), Tiruchirappalli -15 , India. He received MCA degree from Anna University, Tamil $\mathrm{Nadu}$, India. His research interests include the areas of wireless ad-hoc and wireless sensor networks. 


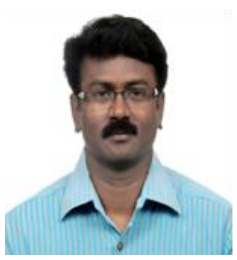

Dr. Alphonse P J A is presently working as an Associate Professor in the Department of Computer Applications, National Institute of Technology, Tiruchirappalli. He did his M.Tech (computer science) in IIT Delhi, India and earned his $\mathrm{PhD}$ from NIT-Tiruchirappalli under Bharathidasan University. His specializations are Graph Theory and its Algorithms, Wireless and Ad-hoc Networks, Fault Tolerant Networks, Cryptography and Network Security.

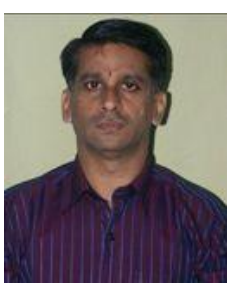

Dr. Janakiraman $\mathbf{T} \mathbf{N}$ is currently working as a Professor in the Department of Mathematics, National Institute of Technology, Tiruchirappalli, India. He has completed his Post graduation at National College, Tiruchirappalli, India in 1983. He did his PhD in Mathematics (Graph Theory and its applications) at Madras University. He was a Postdoctoral Research associate for 1 year (1993-1994) in Madras University. He has two sponsored research projects to his credit and published around 70 papers in refereed National/International journals. His research interests include Pure Graph Theory, Applications of Graph Theory to Fault tolerant networks, Central location problems, Clustering of wired \& wireless ad hoc networks, Clustering of cellular and flexible manufacturing models, Image processing, Graph coding and Graph Algorithms.

How to cite this paper: Sivaraj Chinnasamy, Alphonse P J A, Janakiraman T N, "Self-Load Balanced Clustering Algorithm for Routing in Wireless Sensor Networks", International Journal of Intelligent Systems and Applications(IJISA), Vol.9, No.9, pp.46-57, 2017. DOI: 10.5815/ijisa.2017.09.06 